\title{
SEGURANÇA DO PACIENTE CRÍTICO NA ATENÇÃO MATERNO- INFANTIL NO SISTEMA ÚNICO DE SAÚDE: RELATO DE EXPERIÊNCIA
}

\section{ARTIGO ORIGINAL}

SOUSA, Claudia Maria de ${ }^{1}$

BRASILEIRO, Marislei Espíndula ${ }^{2}$

SOUSA, Claudia Maria de. BRASILEIRO, Marislei Espíndula. Segurança do paciente crítico na atenção Materno-Infantil no Sistema Único de Saúde: Relato de experiência. Revista Científica Multidisciplinar Núcleo do Conhecimento. Ano 04, Ed. 12, Vol. 01, pp. 82-105. Dezembro de 2019. ISSN: 2448-0959, Link de acesso: https://www.nucleodoconhecimento.com.br/saude/seguranca-do-pacientecritico

\section{RESUMO}

Objetivo: Relatar experiência vivenciada por uma enfermeira na assistência hospitalar, materno-infantil a pacientes críticos na interface da atenção secundária com a atenção terciária, contextualizando a segurança do paciente nos processos de trabalho em maternidade pública municipal no Estado de Goiás. Método: Tipo de estudo relato de experiência com vivência, realizado através da observação e descrição dos dados. Resultados: Percebeu-se fragilidade nos processos de trabalho da equipe, sobretudo na transferência destas pacientes para a atenção terciária. Observou-se que se faz necessária a adesão às medidas de segurança do paciente pelos profissionais, bem como refletir sobre a qualidade no atendimento, visto que $o$ fato de necessitar atenção hospitalar mais complexa pode causar nas pacientes

\footnotetext{
${ }^{1}$ Pós-Graduação em Enfermagem em Unidade de Terapia Intensiva, Enfermeira pela Universidade Federal de Goiás (UFG).

${ }^{2}$ Doutora em Ciência da Saúde, Mestre em Enfermagem, Docente do CEEN.
} 
insegurança e medo, fenômenos que estão em desacordo com a segurança da assistência materno-infantil. Considerações finais: Após análise dos resultados constatou-se que é de extrema importância da implementação de todos os protocolos de segurança do paciente na instituição. Este estudo pode influenciar nas condutas dos profissionais e/ou contribuir para que a instituição a nível juntamente com a gestão do Município possa adotar medidas que melhorem a segurança dos pacientes e assim garantir uma assistência com qualidade.

Palavras-chave: Sistema Único de Saúde, segurança do paciente, paciente crítico, atenção materno-infantil.

\section{INTRODUÇÃO}

O Sistema único de Saúde (SUS) é constituído por um conjunto de ações e serviços de saúde sob gestão pública, organizado em rede regionalizada e hierarquizada. Com cobertura em todo o território nacional, com direção única em cada esfera de governo (municipal estadual e federal). Atua de maneira integrada, com base nos princípios da universalidade, da equidade, e da integralidade da atenção, nos diferentes pontos da Rede de Atenção à Saúde (RAS). É estruturado em níveis de complexidade que variam de acordo com a densidade tecnológica (CONNAS, 2011; MENDES, 2011; PAIM, et al, 2011).

Os níveis de atenção à saúde no SUS são arranjos organizados e estruturados nos vários pontos da rede e variam da menor densidade tecnológica para maior, a saber: Atenção Primária à saúde, Atenção Secundária, e a Atenção Terciária ou alta complexidade (MENDES, 2011; PAIM, et al, 2011)

A Atenção secundária é compreendida como serviços e procedimentos de média complexidade, incluindo atenção especializada ambulatorial, serviços de urgência e emergência, assistência hospitalar, apoio diagnóstico e logístico, com densidade tecnológica intermediária. (ERDMANN et al, 2013; BRASIL, 2010). 
$\mathrm{Na}$ atenção terciária ou de alta complexidade tecnológica, realiza-se procedimentos de alto custo, inclui assistência hospitalar (clínica médica e cirúrgica), urgência e emergência, atenção especializada, com apoio logístico, diagnóstico e terapêutico. Os serviços são prestados em hospitais de ensino público federal e em hospitais conveniados com iniciativa privada (PAIM, et al, 2011).

A assistência materna e neonatal possui características particulares, por não serem oriundos de enfermidades, e devido a preocupações que as mulheres têm quando procura assistência, que vão além das questões biológicas, muitas vezes revestidas de muitas emoções. Essa experiência vivenciada pela mulher e pelo recém-nascido pode deixar marcas significativas para os dois, tanto positiva quanto negativa, motivo pelo qual a segurança deve incluir todas as medidas, aquelas aplicada a paciente em geral, assim como as específicas para esse grupo em todas as fases da gestação, parto e puerpério (BRASIL, 2014; MARCOLIN, 2015).

O Brasil apesar de apresentar um PIB (Produto Interno Bruto) na sétima colocação a nível mundial, o índice de mortalidade materna ainda é preocupante, mesmo entre os países em desenvolvimento semelhantes. (BRASIL, 2014).

As mortes maternas podem ser classificas como diretas: as que resultam de causas obstétricas durante a gestação, parto e puerpério, e em decorrência de omissões, intervenções, tratamentos incorretos, ou de uma cadeia de eventos associados a qualquer um desses fatores. As causas indiretas estão relacionas a condições pré existentes, ou que se agravaram pelas alterações fisiológicas inerentes a gravidez (BRASIL, 2014; LIMA et al 2017).

A mortalidade neonatal também está intimamente relacionada com a mortalidade materna já que compartilham muitas causas e determinantes. No entanto a mortalidade neonatal precoce na primeira semana de vida é o fator mais relevante na mortalidade infantil, tendo como causa principal a prematuridade (BRASIL, 2014; LIMA et al 2017). 
Diante de complicações na gestação, parto e puerpério, assim como nascimento prematuro, ou outro problema neonatal, a exemplo a cardiopatia congênita ou a síndrome respiratória aguda do recém nascido pode evoluir para um quadro clínico grave, o que exige tratamento e manejo adequado em tempo hábil.

O Paciente é considerado crítico quando se encontra em risco iminente de perder a vida, ou com comprometimento significativo de órgão ou sistema, assim como aquele que apresenta condições de vulnerabilidade clínica de origem traumática, ou outras causas relacionadas a processos que requeiram intervenção imediata clínica, cirúrgica, gineco-obstétrico ou em saúde mental (BRASIL, 2011).

Para pacientes que apresentam quadro clínico crítico a equipe precisa executar todas as ações e procedimentos aplicando as medidas de segurança, dessa maneira, potencializa as chances de desfechos favoráveis.

A segurança do paciente é um dos temas mais estudados nos últimos anos, devido a sua relevância nos cuidados de saúde. De acordo com a Resolução da Diretoria Colegiada (RDC) ํo 36 de 25 de Julho de 2013, segurança do paciente pode ser definida com a redução a um mínimo aceitável, do risco de dano desnecessário associado à atenção à saúde.

Para Organização Mundial da Saúde (OMS) segurança do paciente corresponde à redução ao mínimo aceitável do risco de dano desnecessário associado ao cuidado de saúde (Brasil, 2014). O Brasil é um dos países que compõe a aliança mundial para a segurança do paciente estabelecida pela OMS (BRASIL, 2014; MARCOLIN, 2015).

O cuidado de saúde no SUS tem se demonstrado uma área particularmente complexa, visto que os ambientes hospitalares são diferentes, em suas diversas especialidades, com recursos, perfil de pacientes e de profissionais que variam muito entre os estabelecimentos de saúde (VICENT E AMALBERTI, 2016).

Diante de todas essas considerações percebeu-se a importância de observar e descrever a segurança do paciente na atenção materno-infantil no Sistema Único de Saúde, através de um relato de experiência. 


\section{OBJETIVO}

Observar e descrever a experiência vivenciada por uma enfermeira no contexto da segurança do paciente crítico na assistência secundária e terciária materno-infantil no sistema único de saúde.

\section{METODOLOGIA}

Trata-se de um estudo do tipo relato de experiência com vivência. Para Fachin (2006) o homem compreendendo o que o cerca passa a desenvolver novas descobertas. $O$ método utilizado foi à observação, fundamental para qualquer área das ciências (FACHIN, 2006). Através dessa técnica, o pesquisador pode desenvolver suas atividades no campo científico (ZAMBELLO, et al, 2018), sem necessariamente interferir no local.

Este relato de experiência também é uma pesquisa de campo, pois os dados aqui descritos correspondem ao local do estudo, processo no qual o pesquisador está articulado diretamente com o espaço fonte (ZAMBELLO, et al, 2018), porém não há utilização de dados de pessoas, o que não implica em desafios éticos, atendendo portanto, a Resolução 466 de 2012.

Após a observação foi realizada a descrição do local de estudo, do fluxo de atendimento de pacientes na instituição, os processos de trabalho da equipe, e a transferência de pacientes considerados críticos da atenção secundária para a atenção terciária em uma Maternidade Pública Municipal no Estado de Goiás. O período de observação foi de Setembro de 2018 a Junho 2019.

Para a discussão dos resultados encontrados, foi realizada pesquisa sistemática na literatura. Foram utilizados como referencial teórico as publicações, portarias, manuais e protocolos do Ministério da Saúde (MS), da Agência Nacional de Vigilância Sanitária (ANVISA), Resoluções de Diretoria Colegiada (RDC) e artigos científicos publicados nas bases de dados Scientific Electronic Library Online (Scielo), Centro de LatinoAmericano do Caribe de informações em Ciências da Saúde (BIRENE), Biblioteca 
Virtual de Saúde (BVS), Literatura Científica e Técnica Latino-Americano e do Caribe em Ciências da Saúde (LILACS), e do portal da Coordenação de Aperfeiçoamento de Pessoal de Nível Superior (CAPS).

\section{RESULTADOS}

Foi observado a instituição, seus processos de trabalho durante período de Setembro de 2018 a Junho 2019, em plantões na maioria das vezes no período diurno, com foco em ampliar os conhecimentos referentes ao processo de segurança do paciente.

\subsection{FILOSOFIA DA INSTITUIÇÃO}

Durante o período de Setembro de 2018 a Junho 2019 pode-se observar que a filosofia da instituição é em atender as demandas da população local e regiões pactuadas na assistência materno-infantil, além disso, é suporte para a Atenção Primária em saúde nessa linha de cuidado e porta de entrada de urgência e emergência em ginecologia, obstetrícia, neonatologia e pediatria.

\subsection{CARACTERÍSTICAS DA INSTITUIÇÃO DO ESTUDO}

Maternidade pública, cadastrada no Conselho Nacional de Estabelecimentos de Saúde, é uma unidade de média complexidade, nível de atenção secundário, realiza atendimento ambulatorial multiprofissional: médico, enfermeiro, psicólogo, assistente social, nutricionista, psicólogo, fonoaudiólogo. Presta serviço de urgência e emergência, assistência hospitalar (clínica e cirúrgica). Apoio diagnóstico: Exames de imagens (ultrassonografia), cardiotocografia (CTG) e exames laboratoriais incluindo colpocitologia oncótica (papanicolau). Procedimentos de complexidade intermediária: inserção de dispositivo intra uterino (DIU).

Dividida no âmbito da assistência em três setores: Ambulatórios e Unidade de Urgência e Emergência, Unidade de Internação e Centro Cirúrgico. Contém serviços administrativos, lavanderia, higienização e limpeza, copa - refeitório. 
A instituição dispõe de serviço de residência médica em ginecologia e obstetrícia, é campo de estágio para acadêmicos de enfermagem, medicina (internato), e para estudantes do curso técnico em enfermagem. Possui: Comissão de Controle de infecção Hospitalar $(\mathrm{CCIH})$ formada por uma médica infectologista uma enfermeira e uma técnica em enfermagem; em fase de implantação o Núcleo de segurança do Paciente (NUSP); regulação de pacientes via sistema de informação; assistência farmacêutica; nutricional, e Plano de Gerenciamento de Resíduos dos Serviços de Saúde (PGRSS).

\subsection{ESTRUTURA FÍSICA}

$\mathrm{Na}$ atenção ambulatorial: São dois consultórios (médico), uma sala para realização de exames de ultrassonografia, uma sala para realização de exame de mamografia, e outra sala para atendimento multiprofissional (psicologia e assistência social.

A Unidade de urgência e emergência: Contém uma sala intitulada de "triagem" onde ocorre o primeiro atendimento da enfermagem (aferição dos sinais vitais e uma breve consulta de enfermagem, quando tem enfermeiro disponível para este setor, ocorre neste mesmo local à realização da CTG; um consultório médico (plantonista staff e residente), uma sala de observação, que também é a sala de administração de medicamentos, de primeiro atendimento e estabilização de pacientes em situação de urgência e emergência.

A Unidade de internação é constituída por 20 leitos: Quatro enfermarias com quatro leitos cada, duas enfermarias com dois leitos cada, uma sala de pré parto com três leitos, uma sala de parto com os equipamentos necessário para a assistência ao parto: Cadeira com perneira de apoio, materiais e insumos essências, materiais de urgência e emergência, um berço aquecido, um fluxômetro de oxigênio $\left(\mathrm{O}_{2}\right)$, um fluxômetro de ar comprimido; uma sala de neonatologia para assistência ao recém nascido; um posto de enfermagem, e a farmácia. 
O centro cirúrgico possui três salas cirúrgicas, porém só duas em funcionamento, não tem sala exclusiva de recuperação pós anestésica. Anexado ao centro cirúrgico encontra-se o Centro de Material e esterilização (CME).

Nas demais dependências da unidade encontram-se a recepção, sala de diretoria: geral, clínica e técnica, sala da coordenação de enfermagem, Comissão de Controle de Infecção Hospitalar, Núcleo de Segurança do Paciente, sala de serviços administrativos: Recursos humanos e faturamento, lavanderia, refeitório, departamento de material e limpeza (DML), repouso dos profissionais e estacionamento para os funcionários.

\subsection{RECURSOS HUMANOS}

Verificou-se que serviço funciona em regime de 24 horas, com escala de oito horas diárias de segunda à sexta, para a maioria do pessoal do administrativo, serviço social, nutrição, psicologia e consultas ambulatoriais, e de doze horas nos períodos diurno e noturno para a maior parte dos profissionais da assistência, da higienização e limpeza, motoristas, copa, e alguns funcionários do administrativo (recepção) lotados em escalas de plantões.

\subsection{CATEGORIAS PROFISSIONAIS E QUANTITATIVOS DE PESSOAL}

Enfermeiros (obstetras, porém a maioria é generalista): Três por plantão sendo um responsável por cada setor da assistência: Unidade de urgência e emergência mais o ambulatório, unidade de internação, e o centro cirúrgico juntamente como o $\mathrm{CME}$. O quantitativo de enfermeiros conta também com uma enfermeira gerente de enfermagem, um enfermeiro gerente do Centro cirúrgico e Centro de Material e Esterilização, uma enfermeira como membro da Comissão de Controle de Infecção, uma enfermeira responsável em parceria com diretoria administrativa pelo Núcleo de Segurança do Paciente, outra enfermeira na coordenação do Plano de Gerenciamento de Resíduos dos Serviços de Saúde (PGRSS), lavanderia e serviço de higienização.

Profissionais de saúde não-enfermeiros: 
- Médicos: No ambulatório um gineco-obstetra e dois residentes; na assistência (urgência, internação e centro cirúrgico): um staff gineco-obstetra e dois residentes em ginecologia e obstetrícia; um pediatra e um anestesista por plantão.

- Quatro Farmacêuticos, quatro Assistentes social, duas Psicólogas e uma Nutricionista no ambulatório.

Técnicos em enfermagem: Onze em cada plantão de doze horas distribuídos nos setores.

Diretoria: um diretor administrativo, um diretor técnico, um diretor clínico;

O serviço conta ainda com três motoristas; sete recepcionistas; dois condutores (maqueiros); dois vigilantes (segurança) apenas no período noturno; duas assistentes de recursos humanas; uma funcionária que faz faturamento. Quatro pessoas compõem a equipe da lavanderia; dez a forma a equipe da higienização; e seis realizam serviço de copa e refeitório.

\subsection{RECURSOS MATERIAIS}

Medicamentos essenciais básicos, de uso controlado: antibióticos e psicotrópicos.

Equipamentos: desfibrilador, respirador portátil, bala de $\mathrm{O}_{2}$, insumos, dispositivos (cateter de acesso venoso periférico, sonda vesical de demora e de alívio entre outros); três carros de emergência mantidos lacrados e conferidos periodicamente, e sempre que forem abertos para atendimentos de intercorrências, sendo um para cada unidade assistencial. (Urgência e Emergência, Unidade de internação e Centro Cirúrgico).

\subsection{PRINCIPAIS OCORRÊNCIAS NA UNIDADE}

Os quadros clínicos críticos mais comuns em obstetrícia: Síndrome Hipertensiva Específica da Gravidez (SHEG), síndrome de HELP, eclampsia, pré eclampsia, Hidronefrose, abortamento, hemorragia, trabalho de parto prematuro, descolamento 
prematuro de placenta, gravidez ectópica rota. Em neonatologia: cardiopatia congênita, síndrome respiratória do recém nascido e prematuridade. Esses quadros clínicos coincidem com os que estão definidos no manual técnico Gestação de Alto Risco (2010), e com o Manual de Acolhimento e Classificação de Risco em Obstetrícia (2015) ambos do Ministério da Saúde.

\subsection{FLUXO DE ATENDIMENTO NA MATERNIDADE: ACOLHIMENTO, AVALIAÇÃO, ESTABILIZAÇÃO E TRANSFERÊNCIA}

Na unidade de urgência e emergência, não há classificação de risco implementada, após passar pela recepção e ter uma ficha preenchida com os dados pessoais, as pacientes são direcionada para a "triagem", onde são aferido os sinais vitais, e uma breve consulta de enfermagem (quando tem enfermeiro atendendo), pois esse profissional não é disponível em tempo integral neste setor, quando na ausência do enfermeiro o atendimento é realizado pelo técnico em enfermagem que afere apenas os sinais vitais. Após este procedimento as pacientes são orientadas a aguardar que serão chamadas pelo nome e de acordo com a ordem das fichas. Porém quando é identificado sinal de urgência e evidenciado uma situação de emergência, inicia-se o atendimento imediato pela equipe de enfermagem, e em seguida pela equipe médica, a estabilização do quadro clínico quando possível, dependendo da situação acionase o Serviço Móvel de Urgência (SAMU) para transferência para a assistência de alta complexidade. Conforme a política de urgência e emergência (BRASIL, 2013).

Quando é possível a estabilização, mas permanecem sinais de gravidade, a paciente é inserida no sistema de regulação para pleitear uma vaga em unidade de alta complexidade em outro Município. Após um período de tempo que é relativo, e o deferimento da vaga, ocorre à transferência da paciente para a atenção terciária, conforme a Política Nacional de Regulação (BRASIL, 2015; BRASIL, 2008).

\subsection{UNIDADE DE NEONATOLOGIA}

A Maternidade abordada neste estudo é considerada de baixo risco, porém ocorre de nascer criança com condições de vida grave como as portadoras de cardiopatia 
congênita e as que nascem com síndrome respiratória do recém nascido. Diante destas ocorrências é necessário acionar a assistência de alta complexidade em Unidade de Terapia Intensiva Neonatal (UTIN), ou Unidade de Cuidados Intermediários (UCIN). O Recém nascido é cadastrado no cadastro nacional do Sistema Único de Saúde. Após a obtenção do Cartão Nacional de Saúde, o paciente é inserido no sistema de regulação, após estes procedimentos aguarda-se a vaga, que na maioria dos casos não ocorre rápido, demorando entre 24,48 horas ou mais.

Quando a Central de Regulação (que no município em questão é pactuado com Aparecida de Goiânia) concede a vaga é necessário acionar o Serviço Móvel de Urgência (SAMU) de Aparecida de Goiânia para realizar o transporte do recém nascido, o que às vezes resulta em tempo de espera que pode ser significativo, pois este serviço atende outras demandas de urgência da região pactuada, como orienta a Política Nacional de Regulação (BRASIL, 2015; BRASIL, 2008).

\subsection{PERCEPÇÕES NA VIVÊNCIA NA ROTINA DA INSTITUIÇÃO}

Notou-se superlotação da unidade, grande demanda de pacientes do próprio município e regiões: O município tem pactuação com outros próximos, motivo pelo qual recebe pacientes oriundas destes locais. A unidade de urgência e emergências é porta aberta para o acesso à assistência não podendo dispensar nenhuma paciente, mesmo sendo de municípios não pactuados.

Outros fatores que contribui com a grande demanda é falta de classificação de risco, e também a cultura de atuação política dos gestores municipais (prefeito, vereadores e servidores públicos que ocupam cargo de confiança) que determinam o atendimento a todos que procurarem o serviço desconsiderando as condições da unidade e a autonomia dos profissionais de saúde, muitas vezes induzindo os profissionais a adotar condutas desnecessárias, sub valorizando as recomendações na legislação do SUS, como os protocolos de acolhimento e classificação de risco confeccionados pelo Ministério da Saúde. 
Além de ser campo de atuação de estudantes da área médica (residentes e internos) a instituição também recebe estudantes do curso técnico em enfermagem que estão sempre supervisionados e por um professor (enfermeiro exclusivo). Quanto aos estudantes de medicina não observei um coordenador para direcioná-los, muitas vezes ficando um grande número de alunos em um setor.

Infelizmente há pouca atuação do médico staff em todos os setores da maternidade, sendo a maior parte dos atendimentos e dos procedimentos realizados por residentes, incluindo os do primeiro ano de residência. Muita rotatividade no quadro de pessoal dos médicos staffs, um a cada dia da semana.

Também foi observado situação de ocorrência de violência obstétrica: Repreensão por parte de médicos residentes com as pacientes que chegam chorando alto, ou gritando em quadros clínicos de abortamento e trabalho de parto, demora no atendimento médico, foco no tratamento curativo.

Ainda predomina a cultura da equipe de enfermagem chamar os médicos para os atendimentos e intercorrências durante todo o período, o que revela falha na disponibilidade em tempo integral por parte da equipe médica no plantão.

Ausência de enfermeiro no setor de urgência emergência (UE) em tempo integral, a orientação é priorizar a unidade de internação e o Centro cirúrgico, deixando muitas vezes a UE descoberta da supervisão e assistência do enfermeiro. Quando este profissional não se encontra disponível na UE e ocorre intercorrências clínicas em caráter de urgência, o mesmo é chamado pelos técnicos em enfermagem ou pelos funcionários da recepção, o que às vezes demanda tempo, variável importante nos atendimentos de urgência e emergência.

Falta de materiais essenciais como campos para envolver instrumental cirúrgico, lençóis, problemas de lavanderia (no maquinário) implicando em demora para liberação de campo cirúrgico para o $\mathrm{CME}$, e de roupas (unissex) para os profissionais de saúde, falha na manutenção preventiva e corretiva da autoclave, dificultando o processamento dos artigos e campos na etapa da esterilização. 
Quantidade insuficientes de insumos como sabão líquido e papel toalha, serviço de higienização precário executado por profissionais com pouca capacitação.

Resistência por parte dos profissionais de saúde as recomendações sobre segurança do paciente; estrutura precária no ambiente assistencial; quantidade de leitos insuficiente para atender a demanda, espaço físico pequeno, ausência de salas exclusiva para administração de medicamentos, de estabilização, e para a realização da classificação de risco.

Carência de treinamento da equipe para manuseio de equipamentos de emergência como desfibrilador e respirador portátil: Existem os equipamentos nas unidades da instituição, porém não há capacitação e ou treinamento dos profissionais de saúde das categorias médicos e equipe de enfermagem. Bombas de infusão em pouca quantidade e muito antigas.

Falha na comunicação interpessoal em todas as unidades da Instituição, o que acarreta insatisfação por parte das usuárias do serviço, desgaste dos profissionais das diversas categorias que prestam serviço na maternidade e pouca resolução nos problemas de saúde doença da população assistida.

Desmotivação e desgaste da equipe de enfermagem: Sobrecarga de trabalho e de responsabilidade para os enfermeiros, obstáculos e entraves na assistência devido a falhas e deficiência de processos administrativos e recursos tecnológicos, como falta de impressora, rede de internet deficitária acarretando a subutilização dos sistemas de informação, homogenia da equipe médica em relação às condutas e tratamentos instituídos, ausência de respaldo legal para atuação de enfermeiras obstétricas, falta de espaço físico para a implementação da classificação de risco, baixa remuneração principalmente dos técnicos em enfermagem.

Apesar de a observação parecer pessimista, vale ressaltar que o foco dessa vivência e na segurança do paciente. Gostaria de ter colaborado diversas vezes, mas o método do trabalho era apenas observacional, exceto se ocorresse uma situação de emergência, onde certamente iria participar. 


\section{DISCUSSÃO}

\subsection{PRINCIPAIS OCORRÊNCIAS NA UNIDADE QUE AFETARAM A SEGURANÇA DAS PACIENTES}

Sobre os principais quadros clínicos críticos ocorridos na unidade a exemplo a Síndrome Hipertensiva Específica da Gravidez (SHEG), gravidez ectópica, abortamento, hemorragia e Trabalho de Parto prematuro, corrobora com dados que o estudo de Lima et al, (2017), aponta como causas diretas e indiretas de óbito materno no Estado de Goiás.

Quanto à resistência por parte dos profissionais das recomendações sobre segurança do paciente, os estudos dizem que as condutas da equipe devem ser baseadas em conhecimento técnico e evidências científicas (COSTA et al, 2018; PANDOVANE, et al, 2018; ROMERO et al, 2018 e SILVA et al, 2016).

Situação de violência obstétrica não deveria ocorrer na assistência à saúde. Segundo (PADOVANI, et al, (2018) em uma revisão integrativa da literatura sobre determinantes da assistência materno-infantil, o cuidado prestado pela equipe dever respeitoso, digno e humanizado respeitando as queixas da mulher, focando o bem estar da gestante e da família. Outro princípio que deve ser aplicado pelos profissionais de saúde é o da ética visto que as obrigações éticas que permeiam a prevenção e a promoção da segurança do paciente são fundamentadas nos quatro princípios da bioética (ROMERO et al, 2018).

Outro fator que pode afetar a segurança das pacientes é pouca atuação médico staff na unidade de urgência e emergência, visto o entrosamento da equipe é considerado um dos pontos mais importantes na assistência obstétrica segura (PANDOVANE, et al, 2018). 


\subsection{FLUXO DE ATENDIMENTO}

Quanto ao fluxo de atendimento como descrito anteriormente ocorre o acolhimento, avaliação, estabilização e encaminhamento para a atenção terciária pela central de serviço de regulação, e dos pacientes graves através do Serviço Móvel de Urgência (SAMU) conforme (BRASIL, 2013). Porém não possui a sala de estabilização como recomenda a Portaria № 2.338, de 3 de Outubro de 2011, que estabelece diretrizes e cria mecanismos para a implantação do componente Sala de Estabilização (SE) da Rede de Atenção às Urgências.

Dentro da Rede de Urgência e Emergência, a sala de estabilização tem a finalidade de proporcionar condições para que se possa prestar assistência temporária e qualificada para a estabilização de pacientes em estado crítico, para posterior encaminhamento a outros pontos da rede de atenção à saúde. (BRASIL, 2011).

\subsection{PERCEPÇÕES NA VIVÊNCIA NA ROTINA DA INSTITUIÇÃO}

A superlotação da unidade tendo como uma das causas a migração de gestantes de outros municípios assemelha-se a realidade de serviços de assistência ginecológica e obstetrícia no âmbito do SUS no Brasil (Sousa, et al2017). Outras variáveis similares sobre a grande demanda nestes estabelecimentos são: a carência de recursos humanos e materiais no atendimento ao parto na rede pública, o déficit de leitos, e as condições insalubres vivenciadas pelos profissionais de saúde (ASSUNÇÃO, et al 2014).

A Indisponibilidade do enfermeiro na unidade urgência e emergência em tempo integral e a ausência da classificação de risco configuram inconformidade com Política Nacional de Humanização do SUS, e com as recomendações das diretrizes da Rede de atenção as Urgências e Emergências, que preconizam o acolhimento com avaliação e classificação de risco visando à qualidade e a humanização nos cuidados de saúde. 
A classificação de risco é um método de avaliação, uma maneira dinâmica de organizar a demanda com base na necessidade de atendimento, sobretudo nos casos de urgências e emergências. Através desta ferramenta o profissional de saúde avalia e direciona os usuários para a forma de atendimento mais adequado, de acordo com a prioridade clínica, essa estratégia permite que haja impacto significativo em situações agudas, ou crônicas agudizadas graves, que se não tratadas em tempo hábil pode haver um desfecho desfavorável (BRASIL, 2013; BRASIL, 2009).

No âmbito da equipe de enfermagem a classificação de risco e a priorização da assistência em Serviços de Urgência é função privativa do enfermeiro, sendo que este profissional deverá estar dotado de conhecimentos, competência e habilidade para garantir rigor técnico-científico aos procedimentos necessários. (COFEN, 2012)

Devido à falta de estrutura adequada da Maternidade, muitas pacientes são transferidas em ambulâncias do próprio Município, que são pequenas, com pouco espaço, o que pode dificultar a atuação dos profissionais em situações instabilidade da paciente, e algumas vezes faltam equipamentos essenciais como bala de oxigênio.

Outros fatores recorrentes são: Falha na manutenção dos veículos, e remanejamento das ambulâncias por parte da gestão (Secretaria Municipal) para outros pontos de atenção à saúde deixando a maternidade descoberta deste recurso.

Em relação à falta de materiais e insumos básicos essenciais, esse dado corrobora com o estudo de Silva et al (2016) que aponta que as instituições de saúde brasileiras vêm enfrentando fatores que afeta a segurança do paciente, entre eles a falta de insumos básicos, problemas com equipamentos e falha na estrutura física.

Quanto à ausência de treinamentos dos profissionais de saúde com o manuseio dos equipamentos utilizados em situação de urgência emergência, entre as recomendações apontadas na dimensão organizacional e melhoria contínua para melhorar a segurança do pacientes estão à realização de capacitações periódicas, o 
aprimoramento dos treinamentos dos funcionários e a correção de práticas inadequadas no serviço (MINUZZI, et al 2016).

$\mathrm{Na}$ assistência obstétrica o treinamento de equipes multiprofissionais em situações de simulação, utilizando manequins ou atores, é extremamente importante para alcançar desfechos favoráveis, essa metodologia capacita a equipe no manejo de situações emergenciais diminuindo o risco de vida para os pacientes (MARCOLIN, 2015).

Porém treinamentos e capacitações feitos de maneira isolada não são efetivos (Silva et al 2016), considerando que uma equipe bem preparada e bem coordenada pode produzir altos níveis de resultados satisfatórios (Vincent e Amalberti (2016). Sobre o tema cultura de segurança nos sistemas de saúde o Ministério da saúde através da ANVISA orienta processos de educação e treinamentos sobre segurança, para assim a instituição alcançar melhoria de desempenho e alta confiabilidade (BRASIL, 2013).

Sobre comunicação interpessoal, esta é considerada uma das dimensões essências para melhorar a qualidade da assistência em saúde e consequentemente a segurança do paciente, falha na comunicação interpessoal como falta de interação inter e multidisciplinar, o medo de causar conflito entre membros da equipe são sinais de fragilidade no cuidado que pode proporcionar uma assistência insegura, e tem prejudicado a assistência nas instituições de saúde brasileiras. Todos os membros da equipe independente da função que exerce, têm a responsabilidade de falar em prol do paciente. A utilização de uma linguagem clara e estruturada é fundamental para a promoção de uma cultura de segurança nos processos de trabalho (PADOVANI, et al 2018; SILVA, et al 2016; BRASIL, 2017).

A Desmotivação e desgaste da equipe de enfermagem são consequências da desvalorização profissional evidenciada pela ausência de plano de carreira e salário no município, baixa remuneração, fragilidade nos processos de trabalho como a insuficiência de recursos necessários para prestar uma assistência segura e livre de danos como determina o Código de Ética dos profissionais de enfermagem (COFEN, 2017). 
Essa multiplicidade de fatores identificadas no cotidiano da instituição deste estudo provoca uma reflexão sobre a complexidade do cuidado em saúde, e vai de encontro ao que afirmam Vincent e Amalberti (2016) as vulnerabilidades do sistema, as atitudes pessoais, a dinâmica de equipe e várias pressões e restrições externas se combinam para produzir um distanciamento em relação às melhores práticas.

\section{CONSIDERAÇÕES FINAIS}

Após análise e discussão dos resultados, pode-se inferir que as medidas de segurança do paciente crítico na assistência materno-infantil não ocorrem de acordo com as recomendações da Política Nacional de Segurança do Paciente. Nesse sentido, vale destacar a importância da implementação dos protocolos de segurança na instituição.

Percebeu-se fragilidade nos processos de trabalho da equipe, sobretudo na transferência destas pacientes para a atenção terciária. Observamos que se faz necessária adesão as medidas de segurança do paciente pelos profissionais, bem como refletir sobre o atendimento humanizado visto que o fato de necessitar atenção hospitalar mais complexa pode causar nos pacientes emoções como insegurança e medo, fenômenos que estão em desacordo com a segurança da assistência maternoinfantil.

Sobre administração pública local, no tocante as políticas de paternalismo para com população e uso do poder político por parte de vereadores, evidencia-se nestas condutas a origem de alguns dos problemas vivenciados pelos profissionais de saúde da instituição, sobretudo na organização do fluxo de atendimentos de pacientes.

No entanto, percebeu-se também pontos positivos, como: Desejo por parte de toda a equipe pela implementação da classificação de risco, o que poderia qualificar a assistência, melhorar assertividade das condutas dos profissionais e da organização do fluxo de pacientes, tornando os processos de trabalho mais efetivos; o NUSP em implementação e fase de Confecção de protocolos assistenciais, que ressaltados na 
literatura no contexto da segurança do paciente a exemplo de estudos (SANT'ANA, et al, 2017; SILVA et al, 2016) e a RDC № 36 de 2013.

Artigos utilizados neste estudo evidenciam que a enfermagem possui papel fundamental na prestação de assistência segura e de qualidade nos diversos cenários dos serviços de saúde (COSTA et al, 2018; PANDOVANE, et al, 2018; SILVA et al, 2016).

Este estudo pode influenciar nas condutas dos profissionais e/ou contribuir para que a instituição possa adotar medidas que melhorem a segurança dos pacientes (mulher e recém-nascido) assim garantir uma assistência com melhor qualidade.

\section{REFERÊNCIAS}

ASSUNÇÃO M.F., SOARES, R. C., SERRANO, I., A superlotação das maternidades em Pernambuco No Contexto Atual Da Política De Saúde. Serv. Soc. Rev., Londrina, v. 16.n.2.p. 05-35, 2014 DOI: 10.5433/1679-4842.2014v16n2p5 Disponível em: http://www.uel.br/revistas/uel/index.php/ssrevista/article/view/14401/15183. Acesso em 17 de Agosto de 2019.

BRASIL. Ministério da Saúde. Agência Nacional de Vigilância Sanitária (ANVISA). Assistência Segura: Uma Reflexão Aplicada à Prática. Série Segurança do Paciente e Qualidade em Serviços de Saúde. Brasília. DF. 2017. Disponível: $\quad$ http://portal.anvisa.gov.br/documents/33852/3507912/Caderno+1++Assist\%C3\%AAncia+Segura++Uma+Reflex\%C3\%A30+Te\%C3\%B3rica+Aplicada+ \%C3\%A0+Pr\%C3\%A1tica/97881798-cea0-4974-9d9b-077528ea1573. Acesso em : 17 de Agosto de 2019.

BRASIL. Ministério da Saúde. Secretaria de Atenção Saúde. Manual de Acolhimento e Classificação de risco em obstetrícia. Brasília. DF 2015. Disponível em:

http://bvsms.saude.gov.br/bvs/publicacoes/documento_referencia_programa_nacion al_seguranca.pdf. Acesso em 10 de Março de 2019. 
BRASIL. Ministério da Saúde. Fundação Oswaldo Cruz. Agência Nacional de Vigilância Sanitária (ANVIA). Documento de Referência para o Programa Nacional de Segurança do Paciente. Brasília. DF. 2014. Disponível em: http://bvsms.saude.gov.br/bvs/publicacoes/documento_referencia_programa_nacion al_seguranca.pdf. Acesso em 14 de Março de 2019.

BRASIL. Ministério da Saúde. Agência Nacional de Vigilância Sanitária . (ANVISA) Serviços de atenção materna e neonatal: Segurança e qualidade. Brasília. 2014. Disponível em: https://proqualis.net/sites/proqualis.net/files/Servi\%C3\%A7os\%20de\%20Aten\%C3\% A7\%C3\%A30\%20Materna\%20e\%20Neonatal\%20\%20Seguran\%C3\%A7a\%20e\%20Qualidade.pdf. Acesso em: 15 de Julho de 2019.

BRASIL, Ministério da Saúde. Secretaria de Atenção à Saúde. Departamento de Atenção Especializada. Manual Instrutivo da Rede de Atenção às Urgências e Emergências no Sistema Único de Saúde (SUS). Brasília. DF. 2013. Disponível em: http://bvsms.saude.gov.br/bvs/publicacoes/manual_instrutivo_rede_atencao_urgenci as.pdf. Acesso em 13 de Agosto de 2019.

BRASIL. Ministério da Saúde . Agência Nacional de Vigilância Sanitária (ANVISA). Resolução da Diretoria Colegiada № 36 de 25 de Julho de 2013. Institui ações para a segurança do paciente em Serviços de Saúde e dá outras providências. Disponível em:

http://portal.anvisa.gov.br/documents/10181/2871504/RDC_36_2013_COMP.pdf/36d 809a4-e5ed-4835-a375-3b3e93d74d5e. Acesso em 15 de Agosto de 2019.

BRASIL. Ministério da Saúde. Secretaria de Atenção à Saúde. Departamento de Atenção Básica. Cadernos de Atenção Básica. Acolhimento a Demanda Espontânea. Queixas mais comuns na Atenção Básica. v. 2. Brasília. DF. 2013. Diponível em: http://bvsms.saude.gov.br/bvs/publicacoes/cadernos_atencao_basica_28.pdf. Acess o em: 17 de Agosto de 2019. 
BRASIL. Ministério da Saúde. Conselho Nacional de Secretários de Saúde CONASS. Coleção para entender a gestão do SUS. Regulação em saúde. v. 10. $1^{\text {a }}$ Ed. 2011. Atualização2015. Disponível em: https://www.conass.org.br/biblioteca/regulacao-em-saude/. Acesso em 16 de Agosto de 2019.

BRASIL. Ministério da Saúde. Conselho Nacional de Secretários de Saúde CONASS. Coleção para entender a gestão do SUS. Sistema Único de Saúde. v. 1. 1. Ed. 2011 Disponível em: https://www.conass.org.br/bibliotecav3/pdfs/colecao2011/livro_1.pdf. Acesso em 14 de Março de 2019.

BRASIL. Ministério da Saúde. Portaria 2.338, de 03 de Outubro de 2011. Estabelece diretrizes e cria mecanismos para implantação do componente sala de estabilização (SE) da Rede de Atenção às Urgências. Disponível em: http://bvsms.saude.gov.br/bvs/saudelegis/gm/2011/prt2338_03_10_2011.html. Acesso em 14 de Março de 2019.

BRASIL. Ministério da Saúde. Portaria 4.279 de dezembro de 2010. Estabelece diretrizes para a organização da Rede de Atenção à Saúde no âmbito do Sistema Único de Saúde (SUS). Disponível em: http://bvsms.saude.gov.br/bvs/saudelegis/gm/2010/prt4279_30_12_2010.html. Acesso em: 17 de Agosto de 2019.

BRASIL. Ministério da Saúde. Secretaria de Atenção à Saúde Departamento de Ações Programáticas Estratégicas. Manual Técnico Gestação de alto risco. Brasília. 2010.2 Disponível em: http://bvsms.saude.gov.br/bvs/publicacoes/gestacao_alto_risco.pdf. Acesso em : 08 de Julho de 2019.

BRASIL. Ministério da Saúde. Secretaria de Atenção à Saúde. Política nacional de Humanização da atenção e Gestão do SUS. Acolhimento e Classificação de Risco nos Serviços de Urgência. Brasília. DF. 2009. Disponível em: 
http://bvsms.saude.gov.br/bvs/publicacoes/acolhimento_classificaao_risco_servico_u rgencia.pdf. Acesso em: 17 de Agosto de 2019.

BRASIL. Ministério da Saúde. Secretaria de Atenção à Saúde. Portaria 1559 de 01 de agosto de 2008. Institui a Política Nacional de Regulação do Sistema Único de Saúde - SUS. Disponível em: http://www.saude.mt.gov.br/upload/legislacao/1559\%5B2870-120110-SES-MT\%5D.pdf. Acesso em: 15 de Agosto de 2019.

COFEN - Conselho Federal de Enfermagem. Resolução 564 de 2017. Aprova o novo Código de Ética dos Profissionais de Enfermagem. Disponível em: http://www.corengo.org.br/resolucao-cofen-5642017_12496.html. Acesso em : 20 de Agosto de 2019.

COFEN - Conselho Federal de Enfermagem. Resolução 423 de 09 de Abril de 2012. Normatiza, no âmbito do Sistema Cofen/Conselhos Regionais de Enfermagem, a Participação do Enfermeiro na Atividade de Classificação de Riscos. Disponível em: http://www.cofen.gov.br/resoluo-cofen-n-4232012_8956.html. Acesso em: 18 de Agosto de 2019.

COSTA, D. B., et al . Cultura de Segurança do Paciente: Avaliação pelos Profissionais de Enfermagem. Texto contexto - enferm., Florianópolis , v. 27, $\mathrm{n}$. 3, e2670016, 2018 . Available from $<$ http://www.scielo.br/scielo.php?script=sci_arttext\&pid=S010407072018000300303\&lng=en\&nrm=iso >. access on 17 Feb. 2019. Epub Aug 06, 2018. http://dx.doi.org/10.1590/0104-070720180002670016.

ERDMANN, A. L., et al . A atenção secundária em saúde: melhores práticas na rede de serviços. Rev. Latino-Am. Enfermagem, Ribeirão Preto , v. 21, n. spe, p. 131139, Feb. 2013 . Available from $<$ http://www.scielo.br/scielo.php?script=sci_arttext\&pid=S0104$11692013000700017 \&$ Ing=en\&nrm=iso >. access on 17 Aug. 2019. http://dx.doi.org/10.1590/S0104-11692013000700017. 
FACHIN, O. Fundamentos da Metodologia. São Paulo: Saraiva, 2006. Disponível: https://www.academia.edu/28917615/Fachin_fundamentos_de_metodologia. Acesso em: 18 de Agosto de 2019.

FONTANA, F. in ZAMBELLO, A. V. et al. Metodologia da Pesquisa e do Trabalho Científico. Capítulo 8- Técnicas de Pesquisa. p. 63. Editora FUNEPE. 2018. Disponível em: http://funepe.edu.br/arquivos/publicacoes/metodologia-pesquisatrabalho-cientifico.pdf. Acesso em 18 de Agosto de 2019.

LIMA, M. R. G., et al . Alterações maternas e desfecho gravídico-puerperal na ocorrência de óbito materno. Cad. saúde colet., Rio de Janeiro , v. 25, n. 3, p. 324331, July $2017 \quad$. Available from <http://www.scielo.br/scielo.php?script=sci_arttext\&pid=S1414462X2017000300324\&Ing=en\&nrm=iso>. access on 14 May 2019. Epub Sep 28, 2017. http://dx.doi.org/10.1590/1414-462x201700030057.

MENDES, E. V. As Redes de Atenção à Saúde. CONASS .OPAS. OMS. Brasília. DF. 2011.2 Disponível em: http://bvsms.saude.gov.br/bvs/publicacoes/redes_de_atencao_saude.pdf. Acesso em: 08 de Julho de 2019.

MARCOLIN, A. C. Qualidade e segurança: caminhos para o sucesso do redesenho do modelo de cuidado obstétrico. Rev. Bras. Ginecol. Obstet., Rio de Janeiro, v. 37, n. 10, p. 441-445, Oct. 2015 . Available from $<$ http://www.scielo.br/scielo.php?script=sci_arttext\&pid=S0100$72032015001000441 \&$ Ing=en\&nrm=iso>. access on 11 May 2019. http://dx.doi.org/10.1590/SO100-720320150005472

MINUZZI, A.P., SALUM N.C., LOCKS, M. O. H., AMANTE, .LN., MATOS, E. Contribuições da equipe de saúde visando à promoção da segurança do paciente no cuidado intensivo. Esc. Anna Nery, Rio de Janeiro , v. 20, n. 1, p. 121129, Mar. 2016. Disponível em: http://www.scielo.br/pdf/ean/v20n1/en_1414-8145ean-20-01-0121.pdf. Acesso em: 14 de Agosto de 2019. 
PADOVANI, C., SILVA, J.P.F., DANTAS, L. S., ANTUNES, M.B., PELLOSO, S. M. Determinantes da Assistência Materno-infantil segura a luz de evidências científicas: Uma revisão integrativa. Revista Nursing. v. 21. n. 247. 2018. p. 2524-2530. Disponível em: http://www.revistanursing.com.br/revistas/247/pg78.pdf. Acesso em 16 de Agosto de 2019.

PAIM J., TRAVASSOS, C., ALMEIDA C., BAHIA, L., MACINKO, J. O sistema de Saúde Brasileiro: História, avanços e desafios. Thelancet. 2011.

Disponível em: https://www.thelancet.com/pb/assets/raw/Lancet/pdfs/brazil/brazilpor1.pdf Acesso em: 06 de Fevereiro de 2019.

ROMERO, M. P.ortela et al . A segurança do paciente, qualidade do atendimento e ética dos sistemas de saúde. Rev. Bioét., Brasília , v. 26, n. 3, p. 333342, Dec. 2018 . Available from $<$ http://www.scielo.br/scielo.php?script=sci_arttext\&pid=S1983-

$80422018000300333 \&$ Ing=en\&nrm=iso $>$. access on 07 June 2019. http://dx.doi.org/10.1590/1983-80422018263252.

SANT'ANA, J. K. A., LEITE, P.O., VILELA, R.P.B., SANFELICE, F.A.N., ALMEIDA, J.B.DE., ALVES, M.C.A. A Importância da Utilização do CHECK LIST de Parto Seguro na Redução de Riscos Obstétricos e Puerperais. Cuidarte. V.11 n.2. DEZ. 2017. p. 300-303. http://www.webfipa.net/facfipa/ner/sumarios/cuidarte/2017v2/300.pdf. Acesso em 13 de Agosto de 2019.

SILVA, A. T., ALVES, M. G. t, SANCHES, R. S., TERRA, F. S., RESCK, Z. M. R. Assistência de enfermagem e o enfoque da segurança do paciente no cenário brasileiro. Saúde Debate. Rio de Janeiro, v. 40, n. 111, p. 292-301, OUT DEZ 2016. Disponível em: http://www.scielo.br/pdf/sdeb/v40n111/0103-1104-sdeb-40-1110292.pdf. Acesso em 14 de Agosto de 2019. 
SOUSA, L. M.O., ARAUJO, E. M., MIRANDA, J. G.V. Caracterização do acesso à assistência ao parto normal na Bahia, Brasil, a partir da teoria dos grafos. Cad. Saúde Pública, Rio de Janeiro , v. 33, n. 12, e00101616, 2017 . Available from $<\mathrm{http}$ ://www.scielo.br/scielo.php?script=sci_arttext\&pid=S0102311X2017001205011\&lng=en\&nrm=iso >. access on 17 Aug. 2019. Epub Dec 18, 2017. http://dx.doi.org/10.1590/0102-311x00101616

VINCENTE , C., AMALBERTI, R., cuidado de Saúde mais Seguro Estratégia Para - Cotidiano do Cuidado. PROQUALIS. Centro Colaborador para a Qualidade do Cuidado e Segurança do Paciente. Rio de Janeiro 2016. Disponível em: https://proqualis.net/sites/proqualis.net/files/Cuidado\%20de\%20Sa\%C3\%BAde\%20 mais\%20Seguro\%20-\%20PDF.pdf. Acesso em : 17 de Agosto de 2019.

Enviado: Setembro, 2019.

Aprovado: Dezembro, 2019. 